\title{
Application of singular perturbation theory in modeling and control of flexible robot arm
}

\author{
Mohit Garg ${ }^{1 *}$, A. Swarup ${ }^{2}$ and A. S. V. Ravi Kanth ${ }^{3}$ \\ M. Tech Student, Department of Electrical Engineering, National Institute of Technology, Kurukshetra, India ${ }^{1}$ \\ Professor, Department of Electrical Engineering, National Institute of Technology, Kurukshetra, India ${ }^{2}$ \\ Associate Professor, Department of Mathematics, National Institute of Technology, Kurukshetra, India ${ }^{3}$
}

\section{(C2016 ACCENTS}

\begin{abstract}
This paper presents dynamic modeling and control for a two-link flexible robot arm. The effort has been made in this paper to explain singular perturbation theory and its application to flexible robot arm. Flexible manipulators have two types of motion, rigid and flexible. Using singular perturbation theory, the system is decomposed into a slow subsystem and a fast subsystem associated with rigid motion and flexible motion respectively under the assumption that the flexible dynamics are faster than the rigid dynamics. Singular perturbation approach is used to drive the slow and fast subsystems with two different time scale subsystems for reduced order modeling. Separate controllers are designed for both slow and fast subsystems. Proportional-derivative (PD) control is used for slow subsystem which tracks the desired trajectory and also ensures the robustness in the controlled system and linear-quadratic regulator $(L Q R)$ is designed for fast subsystem which damps out the vibration of the flexible arm. The model and control of manipulator have been simulated in MATLAB. The dynamic model validation and performance of the system with and without controller are presented.
\end{abstract}

\section{Keywords}

Flexible manipulator, Euler-lagrange approach, Euler-bernoulli beam theory, Assumed-modes method, Singular perturbation theory, PD control, Optimal control method.

\section{Introduction}

Most of the dynamic systems are complex, nonlinear and time varying. There is variety of control techniques for dynamic system studies. Singular perturbation theory is one of the approaches which has been used for analysis and control of dynamic systems [1]. It divides the system dynamics into two parts, slow dynamics and fast dynamics (the solution of the state equation exhibits the phenomenon that some variables move in time faster than other variables, leading to the classification of variables as slow and fast) and thus lessens the dimensionality of the system [2]. The fast dynamics includes the inner loop and slow dynamics forms the outer loop of the feedback system. For each part, control technique is applied separately. Thus, singular perturbation theory is considered as a tool for model order reduction and separation of time scales in control system design. An essential issue in the control system design is the mathematical modeling of a physical system. The modeling of many systems calls for higher order dynamic equations which are very complex to solve mathematically [1].

*Author for correspondence
The presence of some 'parasitic' parameters such as small time constants, resistances, inductances, capacitances, moments of inertia, and Reynolds number, is often the major source for the increased order and "stiffness" of these systems. Singularly perturbed systems or multi-time scale systems often occur generally due to the presence of some parasitic parameters in the mathematical model of the system [3]. The major motivation behind the use of singular perturbation theory to analysis and control of dynamic systems is the alleviation of the high dimensionality and ill-conditioning of the system which causes due to the interaction of slow and fast dynamics.

Singular perturbation theory is used in many applications to control problems. It can be used in aircraft as time scale separation [3] occurs in aircraft models. Pitch rate control dynamics are faster than pitch angle control dynamics. So pitch rate is considered as fast state and pitch angle is considered as slow state. Singular perturbation theory can also be applied in robotics [4]. Flexible manipulators have two types of motion, rigid and flexible. The interaction of rigid and flexible motions in the 
dynamic equations of the flexible manipulators makes the system highly complex and hence it became a challenging and interesting research problem to control such a manipulator while controlling a rigid manipulator is much easier than controlling a flexible manipulator [5]. To rectify the problem associated with the flexible manipulators, singular perturbation theory is adopted as a model reduction tool. With the assumption that the flexible dynamics are faster than the rigid dynamics, singular perturbation theory divides the system into two parts: slow subsystem and fast subsystem associated with rigid motion and flexible motion respectively. The controllers are designed for each subsystem separately. The resulting slow subsystem allows the determination of a tracking control as for rigid manipulators [6] and for the fast subsystem a controller is designed which damps out the vibration of the flexible structure.

The paper is organized in such a way: The mathematical analysis of singularly perturbed systems and two-link flexible manipulator is provided in Section 2 and 3. Section 4 discusses the control of flexible arm by singular perturbation theory. Simulation results are presented and illustrated in Section 5. The final Section presents the conclusion and the further scope of the work.

\section{Singularly perturbed systems}

The systems in which the presence of a small value parameter is mainly responsible for the degeneration (or reduction) of dimension (or order) of the system are named as "singularly perturbed" systems, which are a special class of two-time scale systems. Singularly perturbed systems contain a small value parameter (generally in multiplication with the higher order dynamic state) that cannot be approximated to zero value, unlike the regularly perturbed systems for which an approximation is obtained by simply putting a small value parameter value equal to zero [2].

A dynamic system is described by

$$
\dot{x}=f(x, z, u, t, \varepsilon)
$$$$
\varepsilon \dot{z}=g(x, z, u, t, \varepsilon)
$$

where, $\varepsilon>0$ is a small value scalar quantity, and $x, z$ are slow and fast states respectively and $u$ is control input.

Singular perturbation theory lessens the model order by first neglecting the fast dynamics [1]. It then modifies the considered approximation by reintroducing its effects as boundary layer corrections computed in separate time scales.

For $\varepsilon=0$,

$0=g(\bar{x}, \bar{z}, \bar{u}, t, 0)$

Equation (3) gives us many roots. Putting a root of (3)

$$
\bar{z}=\varphi(\bar{x}, \bar{u}, t)
$$

Now, system given in (1) converted to a reduced model represented as

$$
\dot{\bar{x}}=f(\bar{x}, \varphi(\bar{x}, \bar{u}, t), \bar{u}, t, 0)=\bar{f}(\bar{x}, \bar{u}, t)
$$

Since a small value of perturbation parameter $\varepsilon$ initiates to the fast convergence of $z$ to a root of (3) (which is the equilibrium of (2)), this equation is also called a quasi - steady state model. On the other hand, as (5) deals with the slow dynamic state $x$ this model is also called as the slow model.

Putting $\varepsilon$ to zero, makes the fast variable $z$ instantaneous and there is no guarantee that the fast state $z$ will converge to its quasi - steady state. This convergence must be hold for the validness of our simplified reduced order model.

Since we want to analyze the convergence of the fast variable $\mathrm{z}$ to its quasi-steady state, it is well suitable to shift this quasi-steady state i.e. $\varphi(\bar{x}, \bar{u}, t)$ to the origin.

In order to shift the quasi-steady state of $z$ to the origin, we define $y$ as [7]

$y=z-\varphi(\bar{x}, \bar{u}, t)$

With new time variable $\varepsilon \frac{d y}{d t}=\frac{d y}{d \tau}$, the boundary layer system is obtained as

$$
\frac{d y}{d \tau}=g(t, x, y+\varphi(t, x), 0)
$$

Hence, original singular perturbation problem is formulated into two subsystems: the reduced model and the boundary layer model. The first one is related to the slow state variables while the second one determines the behavior of the fast transients. 


\section{Modeling of two-link flexible manipulator}

A two-link flexible manipulator is considered using two modes of bending deformation for each link whose first link is clamped at its base on the rotor of a motor and second link is loaded with a point mass at its tip as shown in Figure 1.

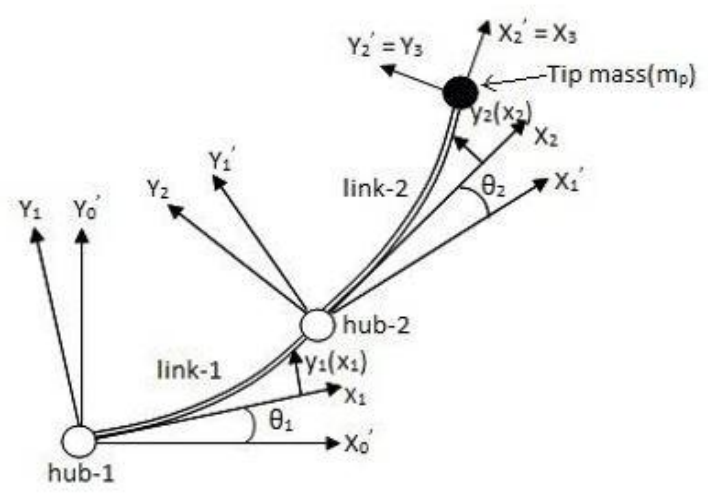

Figure 1 A planar two-link flexible arm [8]

The Euler-Lagrange approach [9] is used to derive the dynamic model of the manipulator. EulerBernoulli Beam approach is adopted to model the flexible links with proper clamped-mass boundary conditions. Partial differential equations (PDE's) obtained through Euler-Bernoulli Beam theory need to be discretized for analysis, simulation and development of controllers. So assumed-modes method (AMM) [6] is used for discretization of infinite dimensional systems (infinite number of natural frequencies and mode shapes). The resultant dynamic model is validated through computer simulation and various boundary conditions are applied at the base and at the end of each link to solve the dynamic model.

The final closed form dynamic equations of motion of the manipulator after algebraic simplifications can be presented as [8]

$$
M(q) \ddot{q}+H(q, \dot{q})+K q=\tau
$$

where $q=\left(\theta_{1}, \theta_{2}, \delta_{11}, \delta_{12}, \delta_{21}, \delta_{22}\right)^{T}, \theta_{1}$ and $\theta_{2}$ are the rotation angles of link-1 and link-2 respectively. $\delta_{11}, \delta_{12}$ are the bending deflections of link-1 and $\delta_{21}, \delta_{22}$ are the bending deflections of link2. $M$ is the positive-definite symmetric inertia matrix, $H$ is the vector of coriolis and centrifugal forces, $K$ is the stiffness matrix and $\tau$ is the torque. Next, the state space model is obtained to make the system analysis simpler and to find the response of the states w.r.t. time by solving the system ordinary differential equations (ODEs). Dynamic model of the two-link flexible manipulator of each coordinate is of 2 nd order, hence for designing state space model, state variables are assumed as

$$
\begin{aligned}
& x_{1}=\theta_{1}, x_{2}=\theta_{2}, x_{3}=\delta_{11}, x_{4}=\delta_{12}, x_{5}=\delta_{21}, x_{6}=\delta_{22}, \\
& x_{7}=\dot{\theta}_{1}, x_{8}=\dot{\theta}_{2}, x_{9}=\dot{\delta}_{11}, x_{10}=\dot{\delta}_{12}, x_{11}=\dot{\delta}_{21}, x_{12}=\dot{\delta}_{22}
\end{aligned}
$$

The state-vector is represented as $x=\left[x_{1}, x_{2}, x_{3}, x_{4}, x_{5}, x_{6}, x_{7}, x_{8}, x_{9}, x_{10}, x_{11}, x_{12}\right]^{T}$

The Two-link flexible manipulator dynamics equations can be rewritten in state space form as

$$
\begin{aligned}
& \ddot{q}=M(q)^{-1}(\tau-h(q, \dot{q})-K q) \\
& \ddot{q}_{6 \times 1}=J_{6 \times 6}(q)\left(\tau_{6 \times 1}-h_{6 \times 1}(q, \dot{q})-K_{6 \times 6} q_{6 \times 1}\right), J=i n v(M)
\end{aligned}
$$

\section{Control of flexible arm using singular perturbation approach}

The design of control for flexible link manipulators is challenging and difficult task because they are underactuated systems (the number of input variables are less than degree of freedom of the system) [9]. This difficulty in control system design is encountered in many problems where both the links and joints are flexible since the actuating torque for each link has to control the flexure of both the link and its corresponding joint. To cope with the problem associated with the flexible manipulators, singular perturbation theory is adopted as a tool for reduced order controller design [5]. Using singular perturbation theory, the higher order complex dynamic system is decomposed into simpler subsystems at different time scales and controllers are designed for each subsystem separately [10].

The dynamic model given in (8) can be reconsidered in singularly perturbed framework as

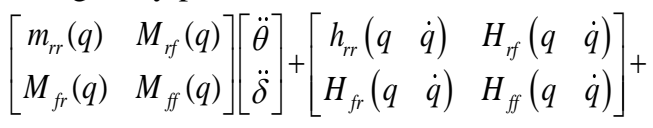

$$
\begin{aligned}
& {\left[\begin{array}{cc}
0 & 0 \\
0 & K_{f f}
\end{array}\right]\left[\begin{array}{l}
\theta \\
\delta
\end{array}\right]=\left[\begin{array}{l}
\tau \\
0
\end{array}\right]}
\end{aligned}
$$

where $r$ and $f$ show rigid and flexible part respectively.

Suppose the singular perturbation parameter ' $\varepsilon$ ' is defined as 
$0<\varepsilon \leq \varepsilon_{\max }=\frac{1}{\sigma\left(K_{f f}\right) \min }$, where $\sigma($.$) min denotes the$ minimum singular value of the concerned variable.

$\tilde{\delta}=\frac{\delta}{\varepsilon} \Rightarrow \delta=\varepsilon \tilde{\delta}, \tilde{K}_{f f}=\varepsilon K_{f f}$

Applying (11) into (10) results,

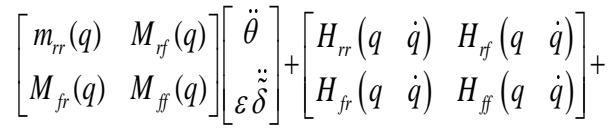

$$
\begin{aligned}
& {\left[\begin{array}{cc}
0 & 0 \\
0 & \tilde{K}_{f f}
\end{array}\right]\left[\begin{array}{l}
\theta \\
\tilde{\delta}
\end{array}\right]=\left[\begin{array}{l}
\tau \\
0
\end{array}\right]}
\end{aligned}
$$

For $\varepsilon=0$, the reduced order model is obtained which defines the rigid dynamics or slow subsystem as

$$
m_{r r}(\theta) \ddot{\theta}+h_{r r}(\theta, \dot{\theta})=\tau_{s}
$$

with $\tilde{\delta}_{o}=-K_{f f}^{-1}\left(B_{f r}(\theta) \ddot{\theta}+H_{f r}(\theta)\right)$.

where $\tau_{s}$ is the slow torque control for slow subsystem and $\tilde{\delta}_{o}$ is the quasi-steady state of fast dynamics.To obtain the fast subsystem, define perturbed variables deviated from slow- manifold as [10]

$$
\xi=\tilde{\delta}-\tilde{\delta}_{o}, z_{1}=\xi, z_{2}=\mu \dot{z}_{1}
$$

where $\mu=\sqrt{\varepsilon}$ is a small constant used for separate time scales.

Using the change of variable method with $p=t / \mu$,

$$
z_{2}=\mu \frac{d z_{1}}{d t}=\frac{d z_{1}}{d p}
$$

By applying (14) and (15) into (12), following equations are obtained.

$$
\frac{d z_{1}}{d p}=z_{2}
$$

$\frac{d z_{2}}{d p}=H_{f f}(\theta) \tilde{K}_{f f} z_{1}-H_{f r}(\theta) \tau_{f}$

where $\tau_{f}$ is the torque control for fast-subsystem.

\subsection{PD control}

The control architecture of PD control is shown in Figure 2. It comprises of a feedforward compensator and a PD feedback control loop.

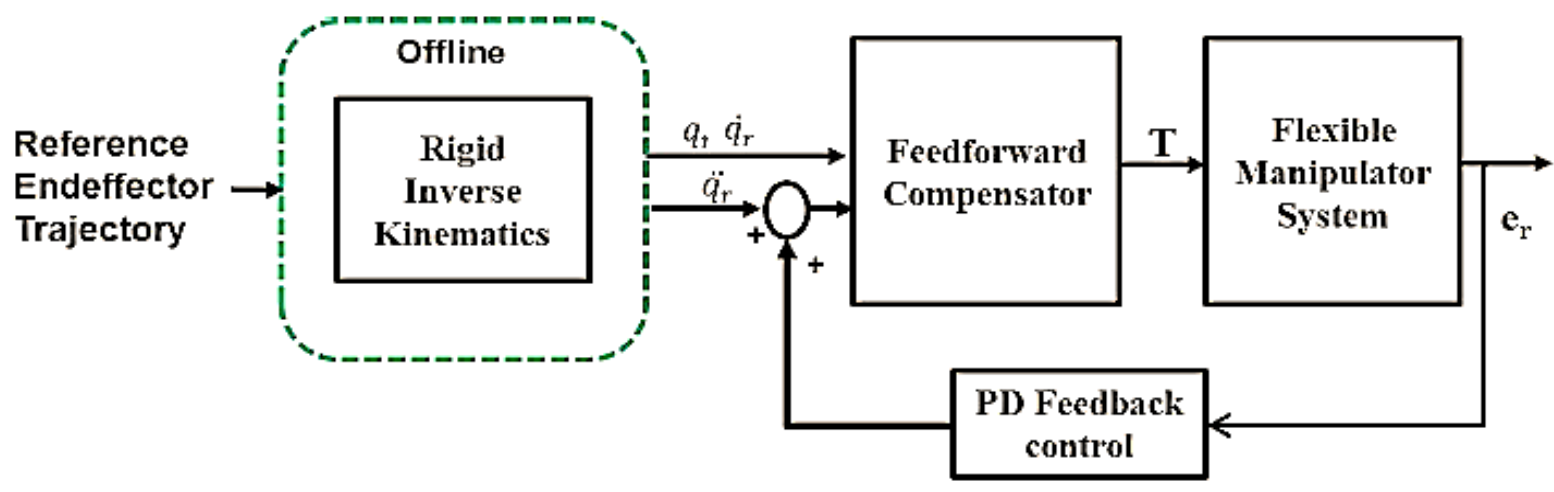

Figure 2 PD control scheme

Using (10), the feedforward compensator is defined as

$$
m_{r r}(\theta) \ddot{\theta}+M_{r f} \ddot{\delta}+h_{r r} \dot{\theta}+h_{r f} \dot{\delta}=\tau_{s}
$$

The coupling effect of rigid body motion and elastic deformations of flexible link can be ignored in the (10) to study the effect of flexibility in control design.

After simplifications, (17) is rewritten as

$$
m_{r r}(\theta) \ddot{\theta}+h_{r r}(\theta, \dot{\theta})=\tau_{s}
$$

A PD feedback control at joint space is designed which is implemented as

179 $m_{r r}\left(\ddot{\theta}+K_{p} e_{r}+K_{v} \dot{e}_{r}\right)+h_{r r}(\theta, \dot{\theta})=\tau_{s}$

Where, $K p$ and $K v$ are the position and velocity error gains, respectively; $e_{r}$ and $\dot{e}_{r}$ are the joint trajectory error at position and velocity level, respectively.

\section{Simulation results}

The dynamic model of the two-link flexible manipulator is simulated using ode45 solver in MATLAB and open-loop responses are presented in Figure 3. The open-loop responses are found to be unstable as dynamic model is highly nonlinear and complex. PD-controller is designed for the system based on Root-locus loop shaping technique. This 
technique involves dominant pole placement (placing open-loop poles at proper places) so that the openloop model also achieves the robustness in the performance.

The physical parameters of the two-link manipulator considered for simulation are given in the Table 1 below.

Table 1 Physical parameters of manipulator [5]

\begin{tabular}{ll}
\hline Parameter & Value \\
\hline (i) Mass density & $\mathrm{p}_{1}=\mathrm{p}_{2}=0.2 \mathrm{~kg} / \mathrm{m}$ \\
$\begin{array}{l}\text { (ii) Length } \\
\text { (iii)Mass of link and }\end{array}$ & $\mathrm{l}_{1}=\mathrm{l}_{2}=0.5 \mathrm{~m}$ \\
payload & $\mathrm{m}_{1}=\mathrm{m}_{2}=\mathrm{m}_{\mathrm{p}}=0.1 \mathrm{~kg}$ \\
(iv) Flexure rigidity & $\mathrm{EI}_{1}=\mathrm{EI}_{2}=1.0 \mathrm{~N}-\mathrm{m}^{2}$ \\
(v) Rotor inertia & $\mathrm{J}_{01}=\mathrm{J}_{02}=0.02 \mathrm{~kg}-\mathrm{m}^{2}$ \\
(vi) Payload inertia & $\mathrm{J}_{\mathrm{p}}=0.005 \mathrm{~kg}-\mathrm{m}^{2}$ \\
\hline
\end{tabular}

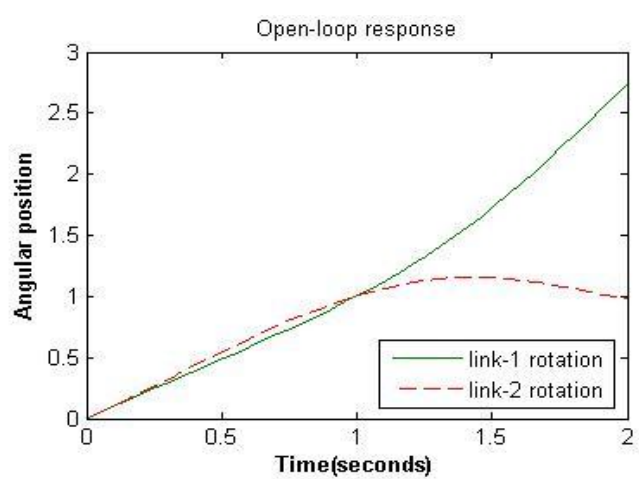

Figure 3 Joint evolution $\left(\theta_{1}(0)=\theta_{2}(0)=0\right)$

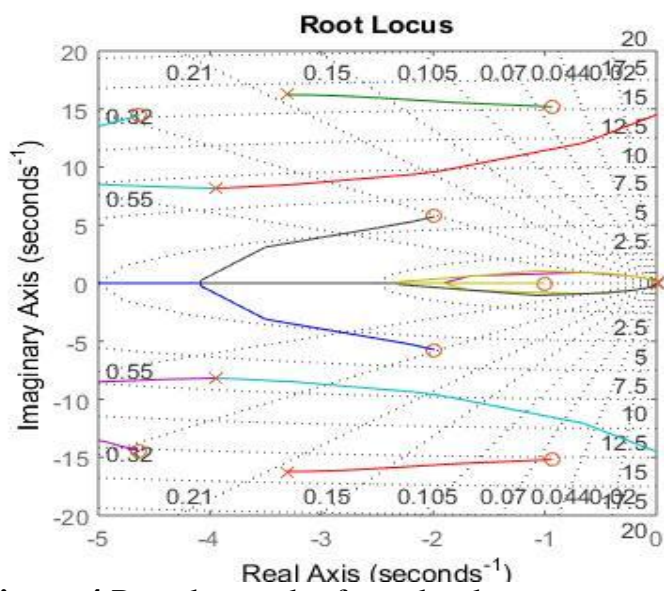

Figure 4 Root-locus plot for pole-placement

From the root-locus as shown in Figure 4, it is observed that for PD controller gains [2.7, 2.7], the system exhibits good stability and less settling time.

The tip position of both the links of the flexible manipulator for PD-controller gains obtained from root locus $(\mathrm{Kp}=\mathrm{Kd}=2.7)$ are plotted in the Figure 5 shown below.

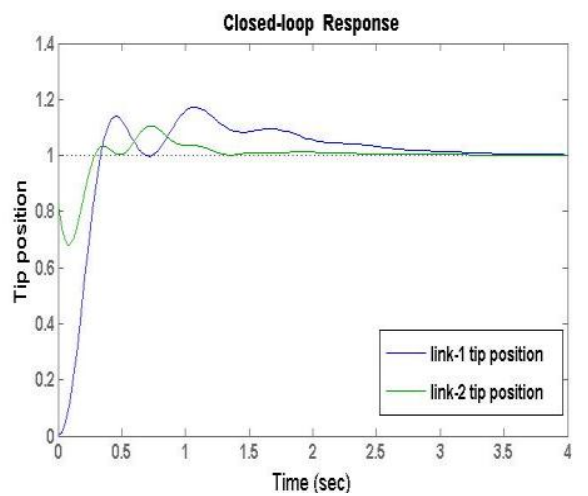

Figure 5 Tip performance using PD- controller

From the above Figure 5, it is observed that good trajectory tracking is achieved through the application of PD control using singular perturbation approach.

\section{Conclusion and future work}

This paper briefly describes the theory of singular perturbation approach and its application to flexible manipulator arm. Since the dynamics of flexible manipulator involves slow and fast states, singular perturbation theory has been proved well useful. The proposed method is useful in modeling and control of systems in which time-scale separation occurs. The open-loop model is validated using mathematical tools. PD-controller is designed for slow subsystem for tracking control and optimal control is used for fast subsystem to damp out the vibrations caused by flexible arm. Nonlinear control can be attempted using Singular perturbation theory. Nonlinear contraction theory can be applied for stability analysis of singularly perturbed systems to converge slow and fast dynamics at a fine convergence rate.

\section{Acknowledgment}

None.

\section{Conflicts of interest}

The authors have no conflicts of interest to declare.

\section{References}

[1] Saksena VR, O'reilly J, Kokotovic PV. Singular perturbations and time-scale methods in control theory: survey 1976-1983. Automatica. 1984; 20(3):273-93.

[2] Kokotovic PV, O'malley RE, Sannuti P. Singular perturbations and order reduction in control theory-an overview. Automatica. 1976; 12(2):123-32. 
[3] Naidu DS, Calise AJ. Singular perturbations and time scales in guidance and control of aerospace systems: a survey. Journal of Guidance, Control, and Dynamics. 2001; 24(6):1057-78.

[4] Zhang Y, Naidu DS, Cai CH, Zou Y. Singular perturbation and time scales in control theories and applications: an overview 2002-2012. International Journal of Information and Systems Sciences.2014; $9(1): 1-36$.

[5] Subudhi B, Morris AS. Dynamic modelling, simulation and control of a manipulator with flexible links and joints. Robotics and Autonomous Systems. 2002; 41(4):257-70.

[6] Kuo KY, Lin J. Fuzzy logic control for flexible link robot arm by singular perturbation approach. Applied Soft Computing. 2002; 2(1):24-38.

[7] Liu Y, Zhu JJ. Singular perturbation analysis for trajectory linearization control. In 2007 American control conference 2007 (pp. 3047-52). IEEE.

[8] De Luca A, Siciliano B. Closed-form dynamic model of planar multilink lightweight robots. IEEE Transactions on Systems, Man, and Cybernetics. 1991; 21(4):826-39.

[9] Dwivedy SK, Eberhard P. Dynamic analysis of flexible manipulators, a literature review. Mechanism and Machine Theory. 2006; 41(7):749-77.

[10] Cheong J, Chung WK, Youm Y. PID composite controller and its tuning for flexible link robots. In IEEE/RSJ international conference on intelligent robots and systems 2002 (pp. 2122-7). IEEE.

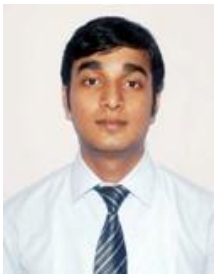

Mohit Garg has graduated in Electronics and Communication Engineering from UPTU Lucknow in 2012. Currenty he is pursuing his M.Tech (Control Systems) in Electrical Engineering Department from National Institute of Technology Kurukshetra. His research interests include robotic control, singular perturbation approach etc.

Email:garg.mohit24@gmail.com

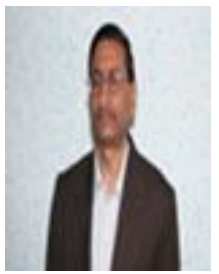

Dr. Akhilesh Swarup received his Ph. D. in 1993 from Indian Institute of Technology (IIT), New Delhi, India. He is currenty working as Professor in the Department of Electrical Engineering at National Institute of Technology (NIT) Kurukshetra, India. $\mathrm{He}$ is a Senior member of IEEE. His research interests include robotics and artificial intelligence, system indentification and control systems.

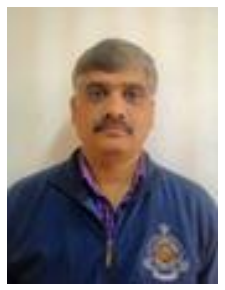

Dr. A. S. V. Ravi Kanth is working as Associate Professor, Head of Department in Mathematics at National Institute of Technology Kurukshetra. He has published more than 40 journal articles in reputed journals. His research interests are numerical analysis of delay diffrential equations, fractional differential equations, singular perturbation problems etc. 